\title{
The Milky Way Halo in Action Space
}

\author{
G. C. Myeong ${ }^{1}$ (D) N. W. Evans ${ }^{1}$, V. Belokurov ${ }^{1}$, J. L. Sanders ${ }^{1}$ (D), and S. E. Koposov ${ }^{1,2}$ (D) \\ ${ }^{1}$ Institute of Astronomy, University of Cambridge, Madingley Road, Cambridge CB3 0HA, UK \\ ${ }^{2}$ McWilliams Center for Cosmology, Department of Physics, Carnegie Mellon University, 5000 Forbes Avenue, Pittsburgh PA 15213, USA \\ Received 2018 February 9; revised 2018 March 2; accepted 2018 March 10; published 2018 March 27
}

\begin{abstract}
We analyze the structure of the local stellar halo of the Milky Way using $~ 60000$ stars with full phase space coordinates extracted from the SDSS-Gaia catalog. We display stars in action space as a function of metallicity in a realistic axisymmetric potential for the Milky Way Galaxy. The metal-rich population is more distended toward high radial action $J_{R}$ as compared to azimuthal or vertical action, $J_{\phi}$ or $J_{z}$. It has a mild prograde rotation $\left(\left\langle v_{\phi}\right\rangle \approx 25 \mathrm{~km} \mathrm{~s}^{-1}\right)$, is radially anisotropic and highly flattened, with axis ratio $q \approx 0.6-0.7$. The metal-poor population is more evenly distributed in all three actions. It has larger prograde rotation $\left(\left\langle v_{\phi}\right\rangle \approx 50 \mathrm{~km} \mathrm{~s}^{-1}\right)$, a mild radial anisotropy, and a roundish morphology $(q \approx 0.9)$. We identify two further components of the halo in action space. There is a high-energy, retrograde component that is only present in the metal-rich stars. This is suggestive of an origin in a retrograde encounter, possibly the one that created the stripped dwarf galaxy nucleus, $\omega$ Centauri. Also visible as a distinct entity in action space is a resonant component, which is flattened and prograde. It extends over a range of metallicities down to $[\mathrm{Fe} / \mathrm{H}] \approx-3$. It has a net outward radial velocity $\left\langle v_{R}\right\rangle \approx 12 \mathrm{~km} \mathrm{~s}^{-1}$ within the solar circle at $|z|<3.5 \mathrm{kpc}$. The existence of resonant stars at such extremely low metallicities has not been seen before.
\end{abstract}

Key words: Galaxy: halo - Galaxy: stellar content - Galaxy: structure

\section{Introduction}

Samples of halo stars near the Sun provide us with accessible documentation on the early history of the Galaxy. But, like medieval palimpsests, the manuscript pages have been overwritten. Dissipative events, such as the assembly of the Galactic disk, and dissipationless processes, such as phasemixing in the time-evolving Galactic potential, make the text difficult to read. Nonetheless, the spatial distribution, kinematics, and chemistry of local halo stars provides us with important evidence on the nature and timescale of events in the early history of the Galaxy, if we could but decode and interpret it.

Actions are invariant under slow changes (e.g., Goldstein 1980). They have often been suggested as the natural coordinates for galactic dynamics (Binney \& Spergel 1982; Binney 1987), in which of course the potential is evolving in time (though possibly not slowly). One of the advances over the last few years has been the development of fast numerical methods to compute actions in general axisymmetric potentials (Binney 2012; Bovy 2015; Sanders \& Binney 2016). For the first time, this allows the study of the local halo in action space using realistic Galactic potentials comprising disks (both stellar and gas), halo, and bulge (McMillan 2017). The only other work known to us that displays the local halo in action space is the pioneering paper of Chiba \& Beers (2000). These authors worked with a much smaller sample of stars $(\sim 1000)$ and out of necessity used a global Stäckel potential as a model of the Galaxy, as their work predated fast numerical action evaluators.

In this Letter, we map the halo stars in action space using a new data set, the SDSS-Gaia catalog. This has six-dimensional phase space information for 62133 halo stars, an order of magnitude larger than previous studies of the local halo. We use this new data set, coupled with the recent advances in action evaluation, to provide new maps of the local halo in action space, which graphically illustrate the dichotomy between the metal-rich and metal-poor stars. We identify two new components of the halo. First, there is a high-energy retrograde component that is limited to the metal-rich stars. Although retrograde stars have been identified before (Majewski et al. 2012; Helmi et al. 2017), we show here that they are largely restricted to metallicities in the range $-1.9<[\mathrm{Fe} / \mathrm{H}]<-1.3$. Second, we provide strong evidence for the existence of a resonant component. It is present across all metallicities, and it has a strong spatial dependence, which satisfies the characteristics of a dynamically induced resonance. This resonance may be linked to a well-known resonance in disk stars, which causes the Hercules Stream. The presence of resonant stars at such low metallicities has not been seen before. We conclude with a discussion of how these findings are related to fundamental events in the Galaxy's early history.

\section{The SDSS-Gaia Catalog}

The SDSS-Gaia catalog was constructed by Sergey Koposov and contains all SDSS stars down to $r=20.5$. The catalog was made by recalibrating the Sloan Digital Sky Survey (SDSS) astrometric solution, and then obtaining proper motions from positions in the Gaia Data Release 1 (DR1) Source catalog (Gaia Collaboration et al. 2016a, 2016b) and their recalibrated positions in SDSS (see, e.g., Deason et al. 2017; de Boer et al. 2018, for more details). The individual SDSS-Gaia proper motions have statistical errors typically $\sim 2 \mathrm{mas} \mathrm{yr}^{-1}$, or $\sim 9.48 D \mathrm{~km} \mathrm{~s}^{-1}$ for a star with heliocentric distance $D \mathrm{kpc}$. We work here mainly with the subsample of main-sequence stars and blue horizontal branch stars (BHBs) with available spectroscopic measurements, in heliocentric distances of $\lesssim 10 \mathrm{kpc}$. The spectroscopic measurements, such as radial velocities and metallicities, are obtained from SDSS DR9 spectroscopy (Ahn et al. 2012) or the crossmatch with LAMOST DR3 (Luo et al. 2015). Finally, 

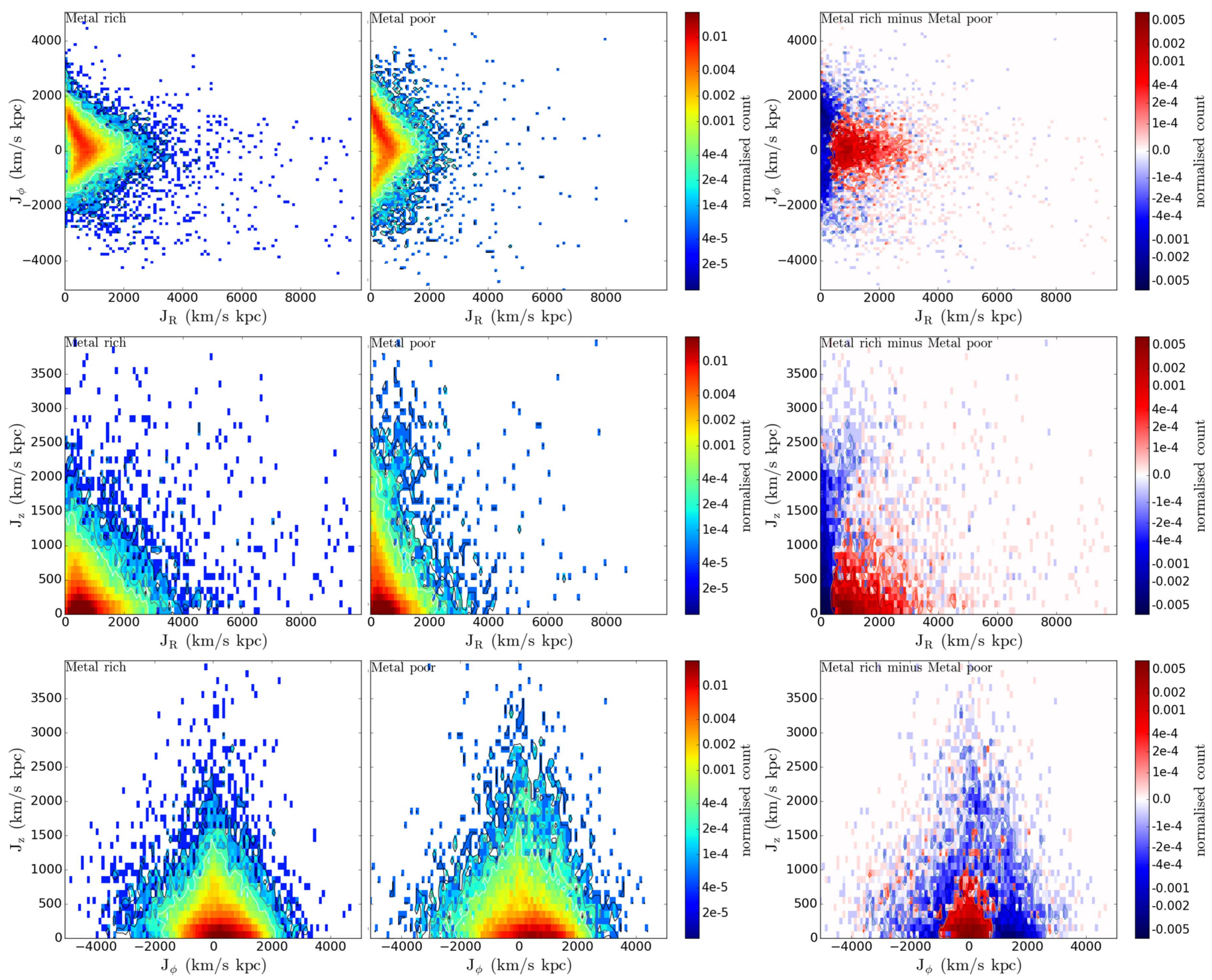

Figure 1. Histograms of the stellar halo in action space $\left(J_{R}, J_{\phi}\right),\left(J_{R}, J_{z}\right)$ and $\left(J_{\phi}, J_{z}\right)$ split into metal-rich (left column, $\left.-1.6<[\mathrm{Fe} / \mathrm{H}]<-1.1\right)$ and metal-poor $($ middle column, $-2.9<[\mathrm{Fe} / \mathrm{H}]<-1.8$ ). The right column displays the difference, with red showing an excess of metal-rich and blue showing an excess of metal-poor stars. Notice (i) the metal-rich stars are tightly clustered around $J_{\phi} \approx 0$ and are much more extended in $J_{R}$, and (ii) the metal-poor stars have prograde rotation $\left(\left\langle J_{\phi}\right\rangle>0\right)$ and a more isotropic distribution in action space.

photometric parallaxes for stars such as main-sequence turnoffs (MSTOs) or BHBs can be added using the formulae in Ivezic et al. (2008) and Deason et al. (2011b) to give samples with the full six-dimensional phase space coordinates.

The SDSS-Gaia catalog currently provides the most extensive catalog of halo stars with positions and kinematics, albeit without an easily calculable selection function. This means that some stellar orbits are not properly represented (such as stars with low- $J_{R}$ and low- $J_{z}$ orbits in small Galactocentric radius), but this will not change the gross morphological features we describe. The actions and energy of each star are estimated using the numerical method of Binney (2012) and Sanders \& Binney (2016) together with the potential of McMillan (2017). The latter also provides the circular speed at the Sun as $232.8 \mathrm{~km} \mathrm{~s}^{-1}$, while for the solar peculiar motion, we use the most recent value from Schönrich et al. (2010), namely $(U, V, W)=(11.1,12.24,7.25) \mathrm{km} \mathrm{s}^{-1}$. Quality cuts and the disk-halo separation employed by Myeong et al. (2018), together with an additional cut on distance error $<2.5 \mathrm{kpc}$, are used to clean the sample. The mode of the distance error is $\sim 0.32 \mathrm{kpc}$. As a result, we obtain a sample of 62133 halo stars (61911 MSTO stars and 222 BHB stars; 59811 stars with SDSS DR9 and 2322 stars with LAMOST DR3 spectroscopy) with full six-dimensional phase space information, actions and energy (see Williams et al. 2017; Myeong et al. 2018, for more details on the cuts and the sample). The metallicity distribution of the halo stars shows evidence of at least two subpopulations (see, e.g., Figure 1 of Myeong et al. 2018). A Gaussian Mixture model from the Scikit-learn (Pedregosa et al. 2011) ${ }^{3}$ based on metallicity suggests the subdivision of the halo sample at $[\mathrm{Fe} / \mathrm{H}] \approx$ -1.67 , with 37670 metal-rich and 24463 metal-poor halo stars.

3 http://scikit-learn.org 


\section{Characteristics of the Halo in Action Space}

\subsection{The Rich and the Poor}

We begin by showing the halo stars in action space $\left(J_{R}, J_{\phi}, J_{z}\right)$. For illustration, we show a metal-rich $(-1.6<[\mathrm{Fe} / \mathrm{H}]<-1.1)$ and metal-poor $(-2.9<[\mathrm{Fe} / \mathrm{H}]<-1.8)$ sample, together with the difference between them in Figure 1. The stratification on linear combinations of the actions suggested by Williams \& Evans (2015) and Posti et al. (2015) is discernible in the triangular shapes of the contours in the left and center panels. However, the metal-rich sample is clearly much more distended toward high $J_{R}$ as compared to $J_{\phi}$ or $J_{z}$. This is most evident in the difference plot in the right-most plot of Figure 1, in which the red is preponderant at large values of $J_{R}$, and at low values of $J_{\phi}$ and $J_{z}$. The presence of abundant high-eccentricity stars indicates that the population is radially anisotropic, while the narrow spread in $J_{z}$ suggests the population is also flattened. In contrast, the metal-poor sample is distributed more equally in all three actions. The blue is preponderant at high $J_{z}$ and reaches out to larger values of $J_{\phi}$ in the right-most column of Figure 1. This suggests it is rounder and has a mild net prograde rotation. Although correlations between kinematics and metallicities of halo stars have been reported before (e.g., Chiba \& Beers 2000; Deason et al. 2011a; Beers et al. 2012; Hattori et al. 2013), our pictures of the halo in action space provide a dramatic illustration of the dichotomy between the metal-rich and metal-poor stars.

Clearer details can be uncovered by slicing the halo into a sequence of smaller metallicity bins, as in Figure 2. For the most metal-rich stars, the contours in $\left(J_{\phi}, E\right)$ space at high energy are noticeably "pointy." There is a tight distribution around $J_{\phi} \approx 0 \mathrm{~km} \mathrm{~s}^{-1} \mathrm{kpc}$, again indicating the presence of many stars moving on nearly radial orbits. In general, the contours in the metal-rich panel $([\mathrm{Fe} / \mathrm{H}]>-1.5)$ are diamondlike in $\left(J_{\phi}, E\right)$ space, while they resemble an up-turned bell for the metal-poor $([\mathrm{Fe} / \mathrm{H}]<-2.1)$. Related to this, the metal-rich sample in $\left(J_{R}, E\right)$ space is skewed strongly toward high $J_{R}$, while in contrast, the metal-poor sample shows much less spread toward high $J_{R}$. In fact, in the more metal-poor panels $(-2.9<[\mathrm{Fe} / \mathrm{H}]<-1.9)$, the bulk of the distribution (colored red) shows the reverse trend of decreasing $J_{R}$ with increasing energy $E$.

The metal-rich stars comprise a radially anisotropic and flattened population. The highest energy and most metal-rich stars are strongly retrograde, but the bulk of the population is at lower energies and shows mild prograde rotation. The metalpoor stars form a rounder population. This is also suggested by the broader distribution in $J_{z}$ in Figure 1. The kinematics are more isotropic, and there is significant prograde rotation. To obtain an idea of the three-dimensional shape, we study their kinematics. Properly speaking, we should decompose the distribution into components in action space, much as Belokurov et al. (2018) do in velocity space. Here, we will simply assume that contamination is mild in the extremal metallicity bins. In the most metal-rich bin, the rotational velocity $\left\langle v_{\phi}\right\rangle=25 \mathrm{~km} \mathrm{~s}^{-1}$, while the velocity dispersion tensor is radially anisotropic with $\left(\sigma_{R}, \sigma_{\phi}, \sigma_{z}\right)=(155,77,88) \mathrm{km} \mathrm{s}^{-1}$. So, the ratio of the horizontal to the vertical components of the kinetic energy tensor is $\approx 4.0$. This is equal to the ratio of horizontal to vertical components of the potential energy tensor via the virial theorem. It can be used to calculate the intrinsic shape, as advocated in Agnello \& Evans (2012). Using their
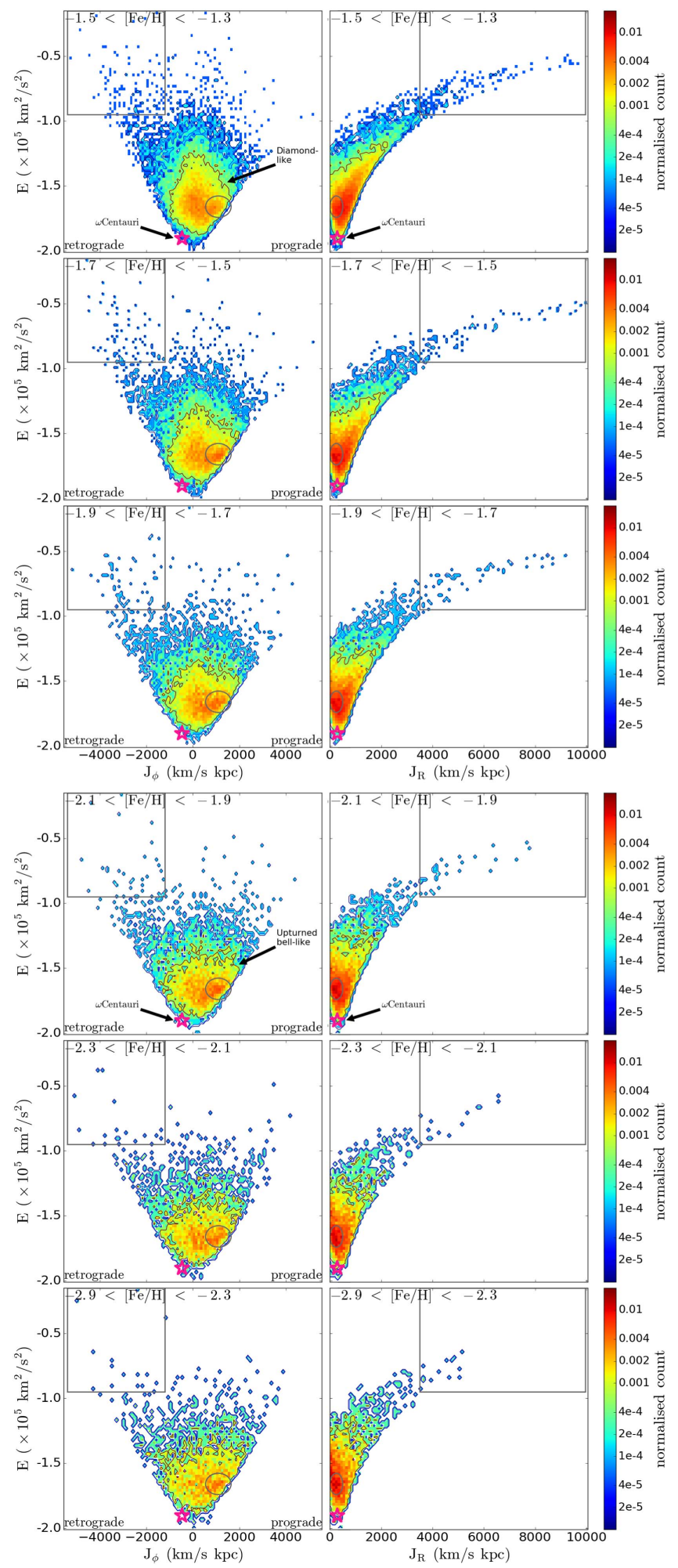

Figure 2. The distribution of halo stars in energy-action space or $\left(J_{\phi}, E\right)$ and $\left(J_{R}, E\right)$ space, split according to six metallicity bins from $-2.9<[\mathrm{Fe} / \mathrm{H}]<$ -1.3 . As we move from the most metal-rich to the most metal-poor, notice that (i) the distribution in radial action becomes more compact as the tail melts away by $[\mathrm{Fe} / \mathrm{H}] \approx-2.0$, (ii) the diamond-like shape of the contours in $\left(J_{\phi}, E\right)$ changes gradually into an up-turned bell-like shape, (iii) the high-energy, retrograde stars (marked by rectangular boxes in the left panels) and the highenergy, eccentric stars (boxes in the right panels) gradually disappear by $[\mathrm{Fe} / \mathrm{H}] \approx-1.9$, and (iv) there is a distinct prograde component at $J_{\phi} \approx 1100$, $J_{R} \approx 150 \mathrm{~km} \mathrm{~s}^{-1} \mathrm{kpc}, E \approx-1.6 \mathrm{~km}^{2} \mathrm{~s}^{-2}$ (marked by ellipses), which is present at all metallicities. The location of $\omega$ Centauri is shown by a pink star. 

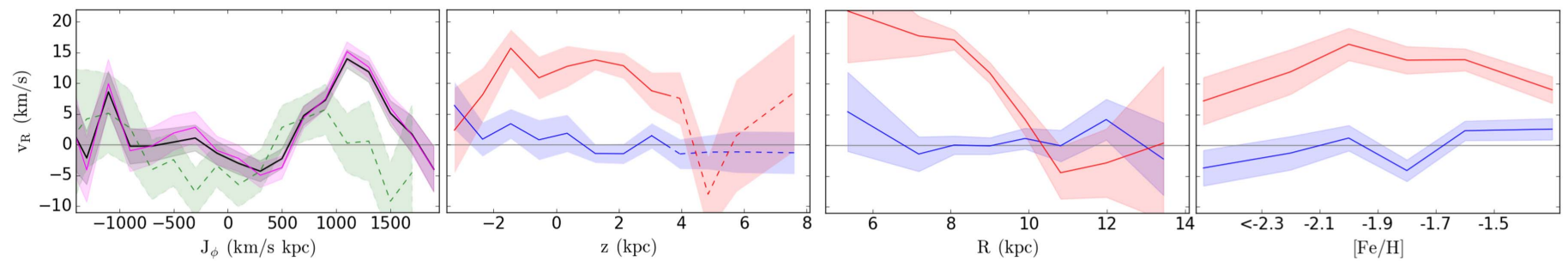

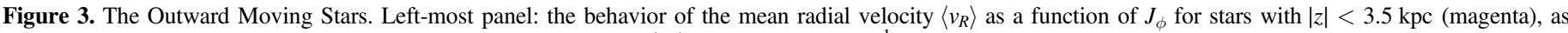

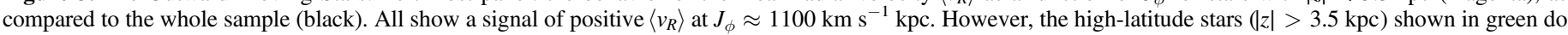

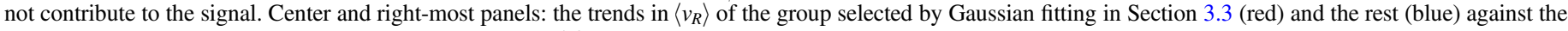

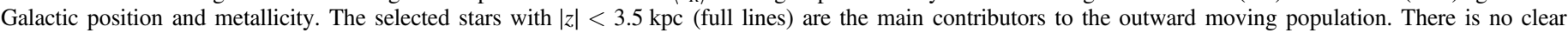

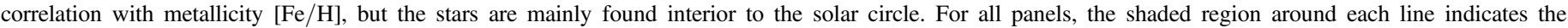
corresponding standard error.

Figure 1, we see that the axis ratio of the density contours of the metal-rich population is $q \approx 0.6-0.7$, depending only modestly on the radial density profile. By contrast, in the most metal-poor bin, the rotational velocity is $\left\langle v_{\phi}\right\rangle=49 \mathrm{~km} \mathrm{~s}^{-1}$, while the velocity dispersion tensor is close to isotropic with $\left(\sigma_{R}, \sigma_{\phi}, \sigma_{z}\right)=(125,114,110) \mathrm{km} \mathrm{s}^{-1}$. This gives an axis ratio for the population of $q \approx 0.9$, so that the density contours are very round. These calculations assume that the total Galactic potential (stars plus dark matter) is spherical. Any flattening in the total potential will result in the computed axis ratios becoming flatter.

\subsection{The Retrograde Stars}

Marked by rectangular boxes in the panels of Figure 2 are the general location of the high-energy (e.g., $E>-1.1 \times 10^{5} \mathrm{~km}^{2} \mathrm{~s}^{-2}$ ), retrograde $\left(J_{\phi}<0\right)$ stars. The box is well-populated in the metalrich panels (e.g., $[\mathrm{Fe} / \mathrm{H}]>-1.9$ ), but sparsely populated in the metal-poor. These metal-rich, high-energy stars have large radial action $J_{R}$ as well, indicating a highly eccentric, retrograde population. This trend diminishes with decreasing metallicity, and the metal-poorer panels (e.g., $[\mathrm{Fe} / \mathrm{H}]<-1.9)$ show a more evenly balanced $J_{\phi}$ distribution of high-energy stars. In addition, irrespective of the sign of $J_{\phi}$, there are noticeably more stars with very high energy (e.g., $E>-0.75 \times 10^{5} \mathrm{~km}^{2} \mathrm{~s}^{-2}$ ) in the metal-rich panels of Figure 2 than the metal-poor. The overdensity of retrograde high-energy stars is evidence of a considerable (retrograde) merger or accretion event in the past (e.g., Quinn \& Goodman 1986; Norris \& Ryan 1989).

\subsection{The Resonant Stars and the Hercules Stream}

The panels also show evidence of a prograde component at around $J_{\phi} \approx 1100, J_{R} \approx 150 \mathrm{~km} \mathrm{~s}^{-1} \mathrm{kpc}, E \approx-1.6 \mathrm{~km}^{2} \mathrm{~s}^{-2}$, which is present at all metallicities as a overdense clump distinct from the general distribution. The component is marked by an ellipse in the panels of Figure 2. We fit a Gaussian with a flat background to isolate this substructure and find a component with mean and dispersion $\left\langle J_{\phi}^{\mathrm{C}}\right\rangle \approx$ $1100 \mathrm{~km} \mathrm{~s}^{-1} \mathrm{kpc}$ and $\sigma_{J_{\phi}}^{\mathrm{C}} \approx 320 \mathrm{~km} \mathrm{~s}^{-1} \mathrm{kpc}$. It is comprised of stars moving on disk-like prograde orbits with intermediate energies. Surprisingly, these stars show a noticeable positive $\left\langle v_{R}^{\mathrm{C}}\right\rangle \approx 12 \mathrm{~km} \mathrm{~s}^{-1}$, so that they have a net outward motion in the Galactic rest frame. This is illustrated in the left-most panel of Figure 3. The positive $\left\langle v_{R}\right\rangle$ signal comes mostly from low Galactic latitudes. The low-latitude stars between $|z|<3.5 \mathrm{kpc}$ (magenta) show positive mean $\left\langle v_{R}\right\rangle$ signatures at the same $J_{\phi}$ range that resembles the signal from the whole sample (black). In contrast, the high-latitude stars with $|z|>3.5 \mathrm{kpc}$ (green) show close to zero mean $\left\langle v_{R}\right\rangle$ across the $J_{\phi}$ range. The magnitude of positive mean $\left\langle v_{R}\right\rangle$ is significant compared to the corresponding standard error.

We select stars with $\left\langle J_{\phi}^{\mathrm{C}}\right\rangle \pm 1.25 \sigma_{J_{\phi}}^{\mathrm{C}}$. The remaining panels of Figure 3 show the $\left\langle v_{R}\right\rangle$ trend of this selected group (red) and the rest (blue). The solid and dashed lines indicate the low- and high-latitude stars for each group (separation at $|z|=3.5 \mathrm{kpc}$ ). Notice the clear difference in the magnitude of $\left\langle v_{R}\right\rangle$ between the selected stars and the rest in various distributions. We also note that the signal drops by an order of magnitude at or near the solar radius and thereafter is close to zero. Interestingly, there is no clear metallicity dependency as the signal remains at a similar magnitude across the metallicity range. Taken together, these facts strongly suggest the source of the signal is dynamical in origin, namely a resonance. This is the first time that a resonance has been identified in such metalpoor stars. The selected stars at low Galactic latitude have $\left(\left\langle v_{R}^{\mathrm{S}}\right\rangle,\left\langle v_{\phi}^{\mathrm{S}}\right\rangle,\left\langle v_{z}^{\mathrm{S}}\right\rangle\right)=(12.2,140.3,3.0) \mathrm{km} \mathrm{s}^{-1}$. These values are indicative, as the selection is crude and probably blended with the other halo populations. However, the value of the mean rotational velocity $\left\langle v_{\phi}^{\mathrm{S}}\right\rangle$ suggests that this component may be associated with the thick disk.

The outward mean radial velocity suggests an association with the Hercules stream, which is also moving outward and is located interior to the solar circle. Stream is a slight misnomer, as the Hercules stream is really a co-moving group of stars of dynamical origin, induced probably by the Outer Lindblad Resonance of the Galactic bar (Dehnen 2000). The Hercules stream has a complex structure with outward $\left\langle v_{R}\right\rangle$ somewhat larger than we measure (Antoja et al. 2014; Hunt et al. 2018), though this is likely accounted by contamination in our sample. It has previously been detected in stars with metallicities $-1.2<[\mathrm{Fe} / \mathrm{H}]<0.4$ (Bensby et al. 2007). However, we see from the panels in Figure 3 that-if our identification with the Hercules stream is correct-then it is detectable right down to $[\mathrm{Fe} / \mathrm{H}] \approx-2.9$ and so is present in stars of metallicity normally associated with thin disk, thick disk, and halo.

\section{Discussion}

There are a number of possible explanations of the properties of the Milky Way stellar halo in action space. The highly flattened metal-rich component is almost certainly the residue of the disruption of accreted dwarf galaxies. The strong radial anisotropy already suggests that the progenitors of this component fell in from large distances. Infall of satellites with random alignments 
tends to isotropize the dispersion tensor. The easiest way to maintain such extreme radial anisotropy is through the infall of one satellite, or group infall of multiple satellites, from a preferred direction (Belokurov et al. 2018). The eccentric halo substructure discovered by Myeong et al. (2017) using the Tycho-Gaia Astrometric Solution (TGAS) crossmatched with RAVE may well be part of this component. The origin of the rounder, metal-poor component is less clear. The isotropic kinematics of this population, together with its roughly spherical shape and mild prograde rotation, are reminiscent of the halo globular clusters. If these objects were once much more massive than the entities surviving today (Schaerer \& Charbonnel 2011), then they may have contributed most of the stars in the metal-poor component of the halo. Alternatively, the accretion of low-mass dwarfs along random directions may have built the metal-poor component. Another contributor could be levitation (Sridhar \& Touma 1996). The growing thin disk can trap stars in the 2:2 resonance and lift them to higher latitude. A pre-existing structure of metal-poor stars could be fattened by such a process.

In addition to these two well-known components, we have identified a high-energy subpopulation that is very strongly retrograde. Helmi et al. (2017) noticed that a high fraction of stars more loosely bound than the Sun are retrograde in the local TGAS sample. Here, we have shown that these stars are overwhelmingly metal-rich, and that the feature does not extend to stars with $[\mathrm{Fe} / \mathrm{H}]$ below -1.9 . A candidate for a retrograde invader exists in the anomalous globular cluster $\omega$ Centauri, long suspected to be the nucleus of a stripped dwarf galaxy (Bekki \& Freeman 2003). It is known to be on a retrograde orbit. Majewski et al. (2012) showed, on the basis of chemodynamical evidence, that it is a major source of retrograde halo stars in the inner Galaxy. The progenitor of $\omega$ Centauri has to be massive, so that the satellite is dragged deep into the potential of the Milky Way and placed on its present low-energy orbit, which is marked by a purple star on Figure 2. While $\omega$ Centauri is on an eccentric orbit, it is considerably retrograde as well, given its present-day energy. Estimates of its initial mass are typically $\sim 10^{10} M_{\odot}$ (Tsuchiya et al. 2003; Valcarce \& Catelan 2011), while its present-day mass is only $5 \times 10^{6} M_{\odot}$ (Meylan et al. 1995). Its disruption must have sprayed high-energy retrograde stars throughout the inner Galaxy, even though it is on a lowenergy orbit now.

Finally, action space has allowed us to trace a new, resonant component down to very low metallicities. This component is also flattened and has prograde rotation with a mean of $\left\langle v_{\phi}\right\rangle \approx 140 \mathrm{~km} \mathrm{~s}^{-1}$. Its most unusual feature is a small, but statistically significant, outward mean radial velocity $\left(\left\langle v_{R}\right\rangle \approx 12 \mathrm{~km} \mathrm{~s}^{-1}\right)$. This is present across the swathe of metallicities in our sample, namely $-2.9<[\mathrm{Fe} / \mathrm{H}]<-1.3$, yet is spatially restricted to just within the solar circle and at $|z|<3.5 \mathrm{kpc}$. The confinement of this prograde feature to a range in Galactocentric radii is unusual and strongly supports a resonance origin. A link with the Hercules stream in disk stars (e.g., Hunt et al. 2018) seems likely.

There are a number of ways the forthcoming Gaia Data Release 2 (DR2) can confirm our picture. First, the crossmatches with radial velocity surveys will have well-defined selection functions. This will allow use of halo distribution functions (Posti et al. 2015; Williams \& Evans 2015) to separate the populations in action space as a function of metallicity and hence better characterize their properties. Second, the SDSS-Gaia catalog undersamples low-latitude stars and so coverage of such substructures is patchy. With Gaia DR2, stars with kinematics akin to the Hercules stream can be traced as a function of location throughout the Galaxy. Third, Gaia colors, as well as spectroscopic follow-up to obtain alpha-abundances, may confirm the origin of the halo components. For example, if the high-energy, retrograde component comes from $\omega$ Centauri, the stripped stars in action space must be trackable to their present position through chemodynamical data. Fourthly, the improved proper motions may even permit the use of angles (as opposed to actions) in studying resonances throughout the halo.

G.C.M. thanks the Boustany Foundation, Cambridge Commonwealth, European \& International Trust and Isaac Newton Studentship for their support of his work. J.L.S. thanks the Science and Technology Facilities Council for financial support. The research leading to these results has received partial support from the European Research Council under the European Union's Seventh Framework Programme (FP/2007-2013) / ERC Grant agreement No. 308024. This work has made use of data from the European Space Agency (ESA) mission Gaia (https://www. cosmos.esa.int/gaia), processed by the Gaia Data Processing and Analysis Consortium (DPAC, https://www.cosmos.esa.int/web/ gaia/dpac/consortium). Funding for the DPAC has been provided by national institutions, in particular the institutions participating in the Gaia Multilateral Agreement.

\section{ORCID iDs}

G. C. Myeong (ib https://orcid.org/0000-0002-5629-8876

J. L. Sanders (iD https://orcid.org/0000-0003-4593-6788

S. E. Koposov (i) https://orcid.org/0000-0003-2644-135X

\section{References}

Agnello, A., \& Evans, N. W. 2012, MNRAS, 422, 1767

Ahn, C. P., Alexandroff, R., Allende, P. C., et al. 2012, ApJS, 203, 21

Antoja, T., Helmi, A., Dehnen, W., et al. 2014, A\&A, 563, A60

Beers, T. C., Carollo, D., Ivezić, Ž., et al. 2012, ApJ, 746, 34

Bekki, K., \& Freeman, K. C. 2003, MNRAS, 346, L11

Belokurov, V., Erkal, D., Evans, N. W., Koposov, S. E., \& Deason, A. J. 2018, arXiv: 1802.03414

Bensby, T., Oey, M. S., Feltzing, S., \& Gustafsson, B. 2007, ApJL, 655 , L89

Binney, J. 1987, in Proc. of the NATO Advanced Study Institute, The Galaxy, ed. G. Gilmore \& B. Carswell (Dordrecht: Reidel), 399

Binney, J. 2012, MNRAS, 426, 1324

Binney, J., \& Spergel, D. 1982, ApJ, 252, 308

Bovy, J. 2015, ApJS, 216, 29

Chiba, M., \& Beers, T. C. 2000, AJ, 119, 2843

Deason, A. J., Belokurov, V., \& Evans, N. W. 2011a, MNRAS, 411, 1480

Deason, A. J., Belokurov, V., \& Evans, N. W. 2011b, MNRAS, 416, 2903

Deason, A. J., Belokurov, V., Koposov, S. E., et al. 2017, arXiv:1703.09230

de Boer, T. J. L., Belokurov, V., \& Koposov, S. E. 2018, MNRAS, 473, 647 Dehnen, W. 2000, AJ, 119, 800

Gaia Collaboration, Brown, A. G. A., Vallenari, A., et al. 2016a, A\&A, 595, A2

Gaia Collaboration, Prusti, T., de Bruijne, J. H. J., et al. 2016b, A\&A, 595, A1 Goldstein, H. 1980, Classical Mechanics (Reading, MA: Addison-Wesley)

Hattori, K., Yoshii, Y., Beers, T. C., Carollo, D., \& Lee, Y. S. 2013, ApJL, 763, L17

Helmi, A., Veljanoski, J., Breddels, M. A., Tian, H., \& Sales, L. V. 2017, A\&A, 598, A58

Hunt, J. A. S., Bovy, J., Pérez-Villegas, A., et al. 2018, MNRAS, 474, 95 
Ivezic, Z., Sesar, B., Jurić, M., et al. 2008, ApJ, 684, 287

Luo, A.-L., Zhao, Y.-H., Zhao, G., et al. 2015, RAA, 15, 1095

Majewski, S. R., Nidever, D. L., Smith, V. V., et al. 2012, ApJL, 747, L37

McMillan, P. J. 2017, MNRAS, 465, 76

Meylan, G., Mayor, M., Duquennoy, A., \& Dubath, P. 1995, A\&A, 303, 761

Myeong, G. C., Evans, N. W., Belokurov, V., Amorisco, N., \& Koposov, S. E. 2018, MNRAS, 475, 1537

Myeong, G. C., Evans, N. W., Belokurov, V., Koposov, S. E., \& Sanders, J. L. 2017, MNRAS, 469, L78

Norris, J. E., \& Ryan, S. G. 1989, ApJL, 336, L17

Pedregosa, F., Varoquaux, G., Gramfort, A., et al. 2011, Journal of Machine Learning Research, 12, 2825
Posti, L., Binney, J., Nipoti, C., \& Ciotti, L. 2015, MNRAS, 447, 3060

Quinn, P. J., \& Goodman, J. 1986, ApJ, 309, 472

Sanders, J. L., \& Binney, J. 2015, MNRAS, 447, 2479

Sanders, J. L., \& Binney, J. 2016, MNRAS, 457, 2107

Schaerer, D., \& Charbonnel, C. 2011, MNRAS, 413, 2297

Schönrich, R., Binney, J., \& Dehnen, W. 2010, MNRAS, 403, 1829

Sridhar, S., \& Touma, J. 1996, MNRAS, 279, 1263

Tsuchiya, T., Dinescu, D. I., \& Korchagin, V. I. 2003, ApJL, 589, L29

Valcarce, A. A. R., \& Catelan, M. 2011, A\&A, 533, A120

Williams, A. A., Belokurov, V., Casey, A. R., \& Evans, N. W. 2017, MNRAS, 468, 2359

Williams, A. A., \& Evans, N. W. 2015, MNRAS, 448, 1360 